\title{
Análise da ocorrência dos critérios de Favrot e das comorbidades apresentadas por cães com dermatite atópica atendidos no Hospital Veterinário da Universidade Federal Fluminense*
}

\section{Analysis of the occurrence of Favrot's criteria and comorbidities presented by dogs with atopic dermatitis treated at the Veterinary Hospital of Universidade Federal Fluminense}

\author{
Júlia Zulske Moulin Ribeiro, ${ }^{* *}$ Bianca de Oliveira Martins, ${ }^{* *}$ Marina Ribeiro Hotz, ${ }^{* *}$ Vivian Gomes Ferreira de Almeida, ${ }^{* *}$ \\ Nathalie Costa da Cunha, ${ }^{* * * *}$ Ana Maria Dieckmann*****
}

\begin{abstract}
Resumo
O objetivo desse trabalho foi analisar a ocorrência dos critérios de Favrot em cães diagnosticados com Dermatite Atópica Canina (DAC) no Hospital Veterinário Professor Firmino Mársico Filho da Universidade Federal Fluminense, bem como sua associação com doenças concomitantes. Foram analisadas 2199 fichas propedêuticas com queixas dermatológicas, dos anos de 2016 e 2017. Do total, 165 (7,5\%) animais foram diagnosticados com DAC dos quais 94 (57\%) eram fêmeas e 71 (43\%) machos. Embora os cães mestiços fossem a maioria dos casos (22,4\%), a raça pura mais frequentemente acometida foi a Poodle (12,5\%). Foram identificados $134(81,2 \%)$ cães com cinco ou mais critérios de Favrot, sendo 32,7\% animais com seis critérios, 26,7\% com cinco critérios, $17,0 \%$ com sete critérios e $4,8 \%$ com oito critérios. Enfatiza-se o aparecimento em mais de $90 \%$ dos casos os critérios intradomiciliado, com prurido primário, responsivo à corticoterapia e com bordas das pinas não acometidas. Dos 165 animais avaliados, 115 (69,7\%) eram portadores de uma ou mais afecções concomitantes à DAC, sendo a otite externa a principal (73\%). Considera-se que os critérios de Favrot podem ser aplicados para a triagem dos pacientes com suspeita de dermatite atópica canina atendidos no HUVET-UFF.
\end{abstract}

Palavras chaves: alergia, canina, dermatologia.

\begin{abstract}
The objective of this study was to analyze the occurrence of Favrot criteria in dogs diagnosed with Canine Atopic Dermatitis (CAD) at the Veterinary Hospital Professor Firmino Mársico Filho of Universidade Federal Fluminense, as well as its association with concomitant diseases. A total of 2199 propaedeutic records with dermatological complaints from 2016 and 2017 were analyzed. Of the total, $165(7.5 \%)$ animals were diagnosed with CAD. Among those diagnosed with CAD, 94 (57\%) were females and 71 $(43 \%)$ males. Although crossbreed dogs were the majority of cases $(22.4 \%)$, the purebred dog most frequently affected by CAD was the Poodle (12.5\%). One hundred and thirty four $(81.2 \%)$ animals were identified with five or more Favrot criteria, $32.7 \%$ animals with six criteria, $26.7 \%$ with five criteria, $17 \%$ with seven criteria and $4.8 \%$ with eight criteria. It emphasizes the appearance in more than $90 \%$ of criteria, dog living mostly indoors, alesional pruritus, glucocorticoid-responsive pruritus and nonaffected ear margins. Of the 165 animals evaluated, $115(69.7 \%)$ had one or more conditions concomitant with CAD, with otitis externa being the main one $(73 \%)$. It is considered that the Favrot criteria can be applied for the screening of patients with suspected canine atopic dermatitis treated at HUVET-UFF.
\end{abstract}

Keywords: allergy, canine, dermatology.

\footnotetext{
*Recebido em 2 de junho de 2020 e aceito em 22 de outubro de 2020.

**Universidade Federal Fluminense, Faculdade de Veterinária, Niterói, RJ, Brasil.

***Universidade Federal Fluminense, Faculdade de Veterinária, Programa de Pós-Graduação Medicina Veterinária (Clínica e Reprodução Animal), Niterói, RJ, Brasil. Autor para correspondência: mvviviangomesf@gmail.com.

****Universidade Federal Fluminense, Faculdade de Veterinária, Departamento de saúde coletiva veterinária e saúde pública, Niterói, RJ, Brasil.

*****Universidade Federal Fluminense, Faculdade de Veterinária, Departamento de patologia e clínica veterinária, Niterói, RJ, Brasil.
} 


\section{Introdução}

A espécie canina é uma das poucas espécies capazes de desenvolver doenças alérgicas espontâneas de pele. Entre estas se encontra a dermatite atópica canina (DAC), segundo distúrbio cutâneo alérgico mais comum na espécie (PUCHEUHASTON, 2015).

A DAC é uma doença crônica de etiologia multifatorial e cunho genético que promove inflamação e prurido cutâneo, a partir da produção de imunoglobulinas $\mathrm{E}$ ( $\mathrm{IgE}$ ) dirigidas contra alérgenos ambientais específicos. Além disso, está associada a desordens na barreira tegumentar e falhas na resposta imunológica (SOLOMON et al., 2012).

Por se tratar de uma doença multifacetada, o diagnóstico deve considerar inúmeros aspectos, como histórico, exame clínico, sintomatologia, exclusão de outras dermatopatias pruriginosas e predisposição racial. Sugere-se que os oito critérios de Favrot sejam utilizados associados a análise dos achados clínicos como ferramenta de auxílio diagnóstico (HENSEL et al., 2015). Quando os cães apresentam cinco critérios há uma sensibilidade de $85 \%$ e especificidade de $79 \%$ para diferenciar a DAC de prurido crônico recorrente. A adição de um sexto parâmetro aumenta a especificidade para $89 \%$ mas diminui a sensibilidade para $58 \%$ (OLIVRY et al., 2010). Os critérios são: início dos sinais clínicos antes do três anos de idade; animal intradomiciliado; prurido responsivo a corticoides; prurido primário; interdígitos torácicos acometidos; pavilhões auriculares afetados; orelhas acometidas, mas sem lesão de bordas; região dorso-lombar sem lesões (FAVROT et al., 2010).

Esses critérios foram determinados a partir de um estudo com trinta e quatro dermatologistas veterinários que trabalhavam em 15 países diferentes localizados na Europa, América do Norte e do Sul e Japão (FAVROT et al., 2010). Ou seja, a maioria dos países se concentrava no hemisfério norte do planeta onde o clima que predomina é o temperado. Porém as condições ambientais tropicais podem influenciar na doença, já que a alergia é um distúrbio determinado pelo ecossistema e variações nos fatores de risco e gatilhos. Nos humanos, que vivem em países tropicais, ocorre a coexposição perene a ácaros domésticos, infecções por helminto e picadas de insetos, que são considerados alérgenos (CARABALLO et al., 2016). E os animais que convivem com os humanos também estão sujeitos a essa exposição perene.

Desta forma, o objetivo desse trabalho foi analisar o uso dos critérios de Favrot em cães com suspeita de DAC para confirmação de diagnóstico no Hospital Veterinário Professor Firmino Mársico Filho da Universidade Federal Fluminense (HUVET- UFF), bem como a associação com doenças concomitantes.

\section{Materiais e métodos}

Para a elaboração da pesquisa, foram analisadas as fichas propedêuticas dos anos de 2016 e 2017, de pacientes caninos do HUVET-UFF. Foram selecionadas 2199 fichas por meio da planilha de controles de atendimento. Os critérios de inclusão foram cães com suspeita clínica de afecções dermatológicas. E posteriormente, após análise das fichas dermatológicas, foram selecionadas somente aquelas cujo suspeita diagnóstica era dermatite atópica canina.

A ficha dermatológica do HUVET era composta por quatro folhas com perguntas pré-estabelecidas detalhadas do animal, do manejo e do ambiente em que vive, visando diferenciar as causas das queixas dermatológicas. Dentre as perguntas havia uma coluna com os oito critérios de Favrot para os médicos veterinários assinalarem quais sinais o animal atendido apresentava. Os atendimentos de queixas dermatológicas dos animais desse estudo foram realizados por médicos veterinários residentes do HUVET devidamente treinados e orientados por médicas veterinárias dermatologistas. Ao final da ficha o médico veterinário determina a suspeita diagnóstica. Foi com base nessa da ficha dermatológica que o grupo deste estudo foi selecionado.

As respostas da anamnese, incluindo a declaração da raça do animal, foram de responsabilidade do tutor.

A partir das fichas dos animais com DAC foram montadas planilhas em Excel囚 para a análise dos dados. Foram avaliadas as frequências das variáveis relativas ao exame clínico, histórico e anamnese dos cães relacionadas aos critérios de Favrot. Utilizou-se o teste de Qui-quadrado, com 95\% de nível de significância para a avaliação de associação da ocorrência dos critérios de Favrot e doenças concomitantes. As análises foram realizadas no programa BioEstat 5.0 (AYRES et al., 2007).

\section{Resultados e discussão}

O estudo retrospectivo dos atendimentos realizados no HUVET no período de janeiro de 2016 a dezembro de 2017 mostrou que entre as 2199 fichas de cães com queixa dermatológica havia $165(7,5 \%)$ animais com suspeita diagnosticada de DAC. Embora os diagnósticos tenham sido realizados por diferentes profissionais, o protocolo usado no HUVET permite afiançar que foram realizadas investigações para afastar outras possíveis causas de dermatites pruriginosas antes do diagnóstico conclusivo de DAC. O protocolo realizado no HUVET em casos de queixas dermatológicas incluía o preenchimento de uma ficha propedêutica específica que continha perguntas mais detalhadas e pré-estabelecidas, incluindo avaliação dos critérios de Favrot e determinação das áreas de lesão no animal. Além disso, foi realizado exame citológico de pele e orelhas, tricograma e exames parasitológicos (com fita adesiva, raspado superficial e/ou profundo).

A frequência de diagnóstico de animais com DAC nesse estudo foi inferior ao estudo realizado em Cuiabá, Mato Grosso, que evidenciou a prevalência de dermatite atópica em 10,5\% dos cães (GASPARETTO et al., 2013). A Tabela 1 apresenta as características da população diagnosticada com dermatite atópica canina no HUVET-U̧FF. 
Tabela 1: Características principais da população de cães diagnosticados com dermatite atópica canina $(\mathrm{N}=165)$, no Hospital Veterinário Professor Firmino Marsico Filho, nos anos de 2016 e 2017

\begin{tabular}{lc}
\hline Características & n/N(\%) \\
\hline Fêmeas & $94 / 165(57 \%)$ \\
Machos & $71 / 165(43 \%)$ \\
Cães castrados & $62 / 165(38 \%)$ \\
Cães não castrados & $103 / 165(62 \%)$ \\
Cães sem raça definida & $37 / 165(22 \%)$ \\
Cães de raça & $128 / 165(78 \%)$ \\
Idade & $5.71 \pm 4.00(1 \text { mês a 15 anos) })^{*}$ \\
\hline
\end{tabular}

$\left({ }^{*}\right)$ Média, desvio padrão e amplitude.

A manifestação da dermatite atópica foi observada em 94 (57\%) fêmeas e 71 (43\%) machos, com diferença estatística entre eles $(p=0,0154)$. Apesar da ausência de estudos confirmatórios sobre a predisposição sexual na $\mathrm{DAC}$, há relatos de maior ocorrência em fêmeas (GRIFFIN; DEBOER, 2001; ZUR et al., 2002) que reforçam o presente resultado assim como há autores que não encontraram diferença significativa entre os números de machos e fêmeas acometidos pela afecção (ZANON et al., 2008).

No presente estudo, $53(56,4 \%)$ cadelas não eram castradas. As principais funções do estrogênio no organismo são a indução da proliferação de células endometriais e o crescimento dos tecidos relacionados à reprodução. Em relação à pele, o estrogênio estimula a mitose epidérmica, modificando a espessura, podendo causar atrofia da camada mais externa da pele (SCOTT, 1996). Essa alteração na epiderme pode prejudicar a função de barreira cutânea e permitir a maior entrada de alérgenos percutâneos. Esta barreira já é naturalmente deteriorada em animais com dermatite atópica (NISHIFUJI, 2014). Observações empíricas demonstram que alterações hormonais do ciclo estral das cadelas podem contribuir para a manifestação ou para o agravamento da dermatite atópica.

A progesterona tem como efeito mais importante no sistema imune, sua ação sobre os linfócitos $T$, favorecendo a secreção de citocinas tipo Th2, enquanto inibe as citocinas Th1 (SZEKERESBARTHO et al., 2005). Na DAC, o sistema imunológico ao apresentar alérgenos ligados à lgE estimula as células de Langerhans a ampliarem a resposta das células Th2. A resposta Th2 também é favorecida pela exposição ao alérgeno na presença de dano na barreira da pele (ONOUE et al., 2009). Essas duas ações combinadas possivelmente provocam um agravamento da manifestação clínica da DAC em fêmeas.

Em estudo realizado com oito cadelas saudáveis observou-se redução nos níveis séricos de $\mathrm{IgE}$ total durante a prenhez, fase em que a progesterona está em alta concentração. Entretanto, os resultados de um teste de dosagem sérica de IgE alérgenoespecífica não foram significativos. Esse teste é utilizado para a determinação do status de sensibilização de animais com DAC (TARPATAKI et al., 2018). Não há estudos semelhantes em cadelas atópicas.
Entre os cães diagnosticados com DAC, $37(22,4 \%)$ eram sem raça definida (SRD) enquanto $128(77,6 \%)$ eram de raça, com diferença estatística $(p<0,0001)$ entre elas. A frequência de cães de raça com DAC no presente estudo foi superior ao encontrado em João Pessoa, Paraíba, onde 6/20 (30\%) dos cães afetados eram SRD e 14/20 (70\%) tinham raça definida. (VASCONCELOS et al., 2020).

A raça de maior frequência foi Poodle com 16 animais (12,5\%); seguida por Shih-tzu com 14 (10,9\%); Yorkshire 13 (10,1\%); Dachshund e Pitbull sete animais (5,4\%), cada. Em estudos realizados em Salvador e São Paulo os cães de raça foram mais acometidos e as raças com maior prevalência de DAC foram Poodle, Maltês, Dachshund e Labrador (SOUZA et al., 2009; RONDELLI et al., 2012; SANTOS;SANTOS, 2016). Já em um estudo realizado na Paraíba as raças Pinscher e Pitbull foram as que apareceram com maior número de cães (VASCONCELOS et al., 2020).

Algumas raças como West Highland White Terrier, Boxer e os Bulldogs Inglês e Francês parecem ser acometidas pela DAC mundialmente enquanto outras como Pastor Alemão, Golden Retriever, Labrador e Dálmata parecem estar predispostas a DAC apenas em algumas regiões geográficas (SHAW et al., 2004; FAVROT, 2014; ANTURANIEMI et al., 2017). A raça é um dado importante, pois já foi determinado que a herança genética é um fator de risco para essa afecção (RONDELLI et al., 2012; FAVROT, 2014; SANTOS;SANTOS, 2016). Embora no HUVET da UFF tenha alta casuística de atendimento a cães SRD, aqueles de raça foram significativamente mais diagnosticados com DAC.

No presente trabalho foi identificado que $134(81,2 \%)$ dos animais portavam cinco ou mais critérios de Favrot. Assim divididos: $44(26,7 \%)$ demonstraram cinco dos critérios, 54 $(32,7 \%)$ seis critérios, $28(17,0 \%)$ sete critérios e $8(4,8 \%)$ oito critérios (Figura 1).

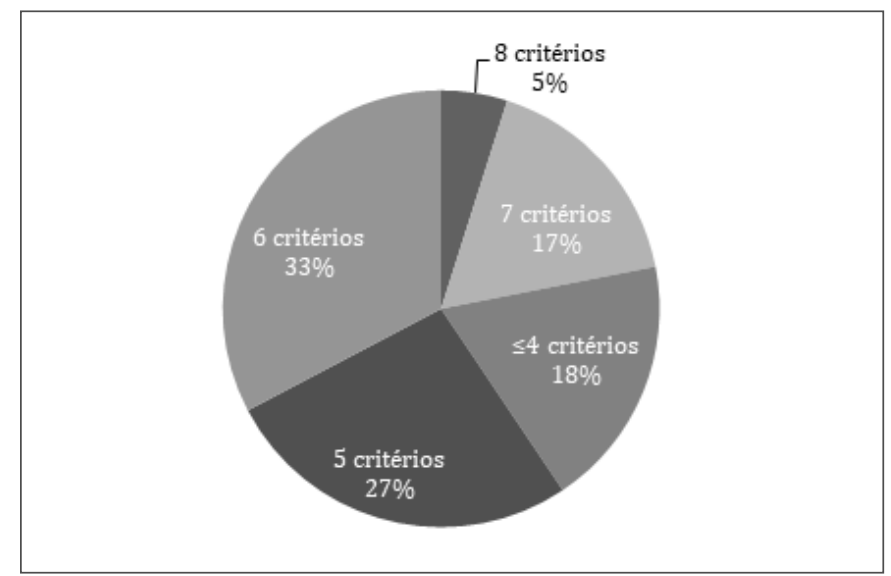

Figura 1: Frequência percentual dos cães que possuíam quatro ou menos, cinco, seis, sete e oito critérios de Favrot $(n=165)$, atendidos no Hospital Veterinário Professor Firmino Marsico Filho, nos anos de 2016 e 2017.

Segundo Hensel et al. (2015) a especificidade dessa ferramenta de auxílio diagnóstico pode ser melhorada dramaticamente, 
aumentando o número de 5 para 6 critérios de Favrot. Quanto mais critérios o animal apresentar, maior as chances de ele ser portador da DAC. Na Tabela 2 estão apresentadas as frequências de cada critério de Favrot.

Tabela 2: Frequência dos critérios de Favrot nos cães diagnosticados com dermatite atópica canina, no Hospital Veterinário Professor Firmino Marsico Filho, nos anos de 2016 e 2017

\begin{tabular}{lcc}
\hline Parâmetros & n/N (\%) & p-valor \\
\hline Intradomiciliado & $160 / 165(97.0 \%)^{\mathrm{a}}$ & $<0.0001$ \\
Prurido Primário & $156 / 165(94.6 \%)^{\mathrm{a}}$ & $<0.0001$ \\
Responsivo à corticoterapia & $59 / 63(93.7 \%)^{\mathrm{a}}$ & $<0.0001$ \\
Bordas das pinas não acometidas & $152 / 165(92.1 \%)^{\mathrm{a}}$ & $<0.0001$ \\
Condutos auditivos acometidos & $131 / 165(79.4 \%)^{\mathrm{b}}$ & $<0.0001$ \\
Interdígitos acometidos & $101 / 165(61.2 \%)^{\mathrm{c}}$ & $<0.0001$ \\
Início sintomas com <3 anos & $82 / 165(49.7 \%)^{\mathrm{d}}$ & 1.000 \\
Dorso-lombar não lesionada & $77 / 165(46.7 \%)^{\mathrm{d}}$ & 0.2286 \\
\hline
\end{tabular}

Letras iguais indicam que não há diferença estatística entre as colunas, pelo teste Qui-quadrado, com 95\% de significância.

Enfatiza-se o aparecimento em mais de $90 \%$ dos casos os critérios intradomiciliado, com prurido primário, responsivo à corticoterapia e com bordas das pinas não acometidas. É importante ressaltar que o dado responsivo a corticoterapia só pôde ser analisado em 63 cães, já que os outros 102 nunca haviam utilizado corticoide ou os tutores não souberam responder. Somente os parâmetros de início antes dos três anos e região dorso-lombar não lesionada não tiveram diferença estatística quando comparados com o seu não aparecimento.

Não há um sinal clínico patognomônico da DAC, porém, considera-se o prurido intenso em áreas sem lesão, o primeiro e mais frequente, principalmente quando ocorrem em face, pavilhão auricular, extremidades dos membros, axilas, região inguinal e perianal e áreas de dobras cutâneas (SOLOMON et al., 2012). Segundo Favrot (2014) $82 \%$ dos cães atópicos passam a maior parte do tempo intradomiciliados. Isso sugere que a exposição prolongada a ácaros e demais alérgenos domésticos pode desencadear ou piorar os sinais clínicos.

A idade típica no início da DAC é relatada entre seis meses e três anos (GRIFFIN; DEBOER, 2001). Foi demonstrado que cerca de $80 \%$ dos cães com DAC apresentam sinais clínicos antes dos três anos de idade. Percentual bem mais elevado do que o encontrado no presente estudo onde aproximadamente $50 \%$ dos pacientes manifestaram os sinais enquanto jovens. A idade média dos animais deste estudo foi $5.7 \pm 4.0$ anos, sendo que apenas $38,8 \%$ tinham três anos ou menos o que pode justificar a falta de identificação precoce dos sinais da dermatopatia.

Dos 165 animais avaliados, 115 (69,7\%) eram portadores de uma ou mais afecções concomitantes a DAC. Na Tabela 3 está descrita a associação de cada critério de Favrot com a ocorrência de outras doenças.
Tabela 3: Doenças concomitantes em animais diagnosticados com dermatite atópica canina atendidos no Hospital Veterinário Professor Firmino Marsico Filho, nos anos de 2016 e 2017

\begin{tabular}{lcc}
\hline Parâmetros & $\begin{array}{c}\text { Presença de } \\
\text { outras doenças }\end{array}$ & p-valor \\
\hline Início sintoma > 3 anos & $54 / 82(65,9 \%)$ & \multirow{2}{*}{0.3690} \\
Início sintoma < 3 anos & $61 / 83(73,5 \%)$ & \\
Intradomiciliado & $111 / 160(69,4 \%)$ & \multirow{2}{*}{1.000} \\
Extradomiciliado & $4 / 5(80,0 \%)$ & \\
Responsivo à corticoterapia & $44 / 59(74,6 \%)$ & 0.5668 \\
Não responsivo à corticoterapia & $2 / 4(50,0 \%)$ & \\
Prurido Primário & $114 / 156(73,1 \%)$ & 0.0003 \\
Sem Prurido Primário & $1 / 9(11,1 \%)$ & \\
Interdígitos acometidos & $86 / 101(85,1 \%)$ & $<0.0001$ \\
Sem interdígitos acometidos & $29 / 64(45,3 \%)$ & \\
Condutos auditivos acometidos & $103 / 131(78,6 \%)$ & $<0.0001$ \\
Sem condutos auditivos acometidos & $12 / 34(35,3 \%)$ & \\
Bordas das pinas não acometidas & $107 / 152(70,4 \%)$ & \multirow{2}{*}{0.9989} \\
Bordas das pinas acometidas & $8 / 12(66,7 \%)$ & \\
Dorso-lombar não acometida & $52 / 77(67,5 \%)$ & 0.6929 \\
Dorso-lombar acometida & $63 / 88(71,6 \%)$ & \\
\hline
\end{tabular}

Ressalta-se que prurido primário, interdígitos e condutos auditivos acometidos apresentaram maior frequência de associação com outras doenças, com diferença estatística. O prurido deve estar presente e sua ausência descarta o diagnóstico de DAC (FAVROT et al., 2010). Em decorrência do intenso prurido, é possível observar lambedura excessiva nas regiões de interdígitos, propiciando o desenvolvimento de infecções e lesões secundárias como hiperpigmentação, alopecia, pústulas e liquenificação. Além disso, existe íntima relação com a ocorrência de otite externa e prurido no pavilhão auricular nos animais atópicos. Sendo a otite já considerada parte dos sinais apresentados por um animal atópico (NGO et al., 2018; MEASON-SMITH et al., 2019). Segundo Favrot et al. (2010) em $43 \%$ dos cães atópicos a apresentação inicial foi otite externa.

Ao avaliar a descrição das comorbidades, observou-se que a otite foi a afecção de maior frequência, acometendo 84 (73,0\%) dos cães estudados, seguida de piodermite $35(30,4 \%)$, dermatite alérgica a picada de ectoparasitas (DAPE) $17(14,8 \%)$, foliculite $7(4,3 \%)$ e pododermatite $5(3,5 \%)$, como mostra a Figura 2.

Dois animais apresentaram malasseziose associada enquanto demodicose, dermatofitose, hipersensibilidade alimentar e furunculose ocorreram em apenas um animal cada. Otite, piodermite e malasseziose secundárias, são complicações comuns nos cães com DAC (GROSS et al., 2009; NGO et al., 2018). Sendo assim, os animais com DAC atendidos no HUVET apresentaram as doenças concomitantes esperadas para animais atópicos. 


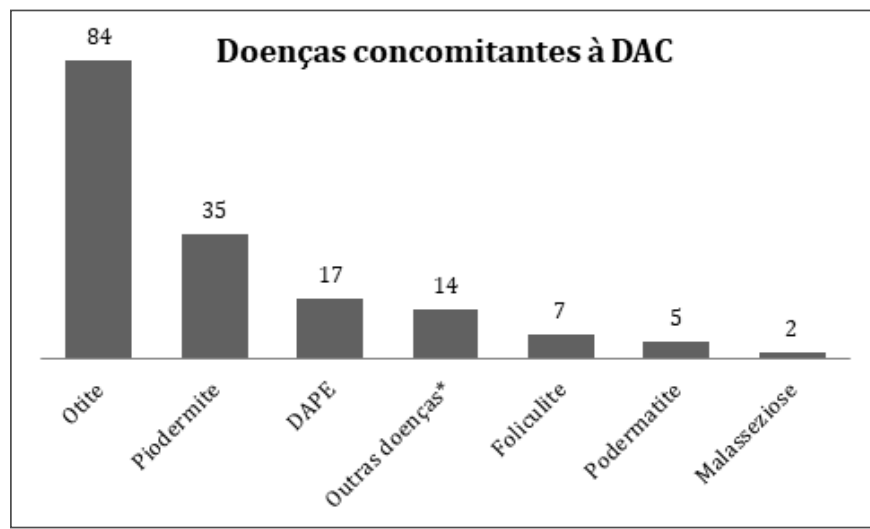

Figura 2: Frequências absolutas de doenças concomitantes em cães diagnosticados com dermatite atópica canina $(n=165)$, no Hospital Veterinário Professor Firmino Marsico Filho, nos anos de 2016 e 2017.

A alta frequência de otites nos cães deste relato corrobora os achados de Zanon et al. (2008) que revelaram que $86 \%$ dos cães atópicos apresentavam otite e é maior do que a encontrada por Favrot et al. (2010) que a observou em apenas $50 \%$ dos pacientes. Sabe-se que a etiologia da otite é multifatorial, porém o prurido repetitivo e a deficiência na barreira cutânea dos indivíduos com dermatite atópica canina são alguns dos

\section{Referências}

ANTURANIEMI, J., UUSITALO, L., HIELM-BJO"RKMAN, A. Environmental and phenotype-related risk factors for ownerreported allergic/atopic skin symptoms and for canine atopic dermatitis verified by veterinarian in a Finnish dog population. PlosOne, v.12, n.6, p.1-17, 2017.

AYRES, M.; AYRES JUNIOR, M.; AYRES, D.L.; SANTOS, A.S. 2007. Bioestat 5.0 - Aplicações estatísticas nas áreas das ciências biomédicas. ONG Mamiraua, Belém, PA. 364p.

CARDOSO, M.J.L.; MACHADO, L.H.A.; MELUSSI, M.; ZAMARIAN, T.P.; CARNIELLI, C.M.; JÚNIOR, J.C.M.F. Archives of Veterinary Science, v.16, n.2, p.66-74, 2011.

CARABALLO, L.; ZAKZUK, J.; LEE B.W.; ACEVEDO, N.; SOH J.Y.; SÁNCHES-BORGES, M.; HOSSNY E.; GARCÍA E.; ROSARIO, N.; ANSOTEGUI, I.; PUERTA, L.; SÁNCHES, J. Particularities of allergy in the Tropics. World Allergy Organization Journal. v.9, n.20, p.1-44, 2016.

FAVROT, C. Clinical signs of canine atopic dermatitis. In: NOLI,C.; FOSTER, A.; ROSENKRANTZ, W. Veterinary Allergy. John Wiley \& Sons, 2014, p. 65-69.

FAVROT, C.; LINEK, M.; MUELLER, R.; ZINI, E. Development of a questionnaire to assess the impact of atopic dermatitis on health-related quality of life of affected dogs and their owners. Veterinary Dermatology, v. 21, p. 64-70, 2010.

GASPARETTO, N.D.; TREVISAN, Y.P.A.; ALMEIDA, N.B.; NEVES, R.C.S.M.; ALMEIDA, A.B.P.F.; DUTRA, V.; COLODEL, E.M.; SOUSA, V.R.F. Prevalência das doenças de pele não neoplásicas em cães no município de Cuiabá, Mato Grosso. Pesquisa Veterinária Brasileira, v.33, 359-362, 2013.

GRIFFIN C.E.; DEBOER D. J. The ACVD task force on canine atopic dermatites (XIV): clinical manifestations of canine atopic dermatitis. Veterinary Immunology and Immunopathology. v.81, p.255-269, 2001. fatores determinantes para o seu aparecimento (NGO et al., 2018; MEASON-SMITH et al., 2019).

Em relação à frequência de DAPE, a percentagem encontrada $(14,8 \%)$ foi inferior aos $50 \%$ observados por Cardoso et al. (2011). Esta diferença se justifica, pois foi observado que 119 $(72,1 \%)$ dos animais faziam controle frequente de ectoparasitas.

\section{Conclusão}

De acordo com os resultados, é importante alertar aos clínicos veterinários de pequenos animais que os critérios de Favrot que mais se repetiram foram cão intradomiciliado, presença de prurido primário, responsivo à corticoterapia e bordas das pinas não acometidas; que a otite concomitante foi frequente sugerindo que se investigue a hipersensibilidade no manejo desta afecção; que o controle cuidadoso de ectoparasitas evita o surgimento da DAPE em paralelo e que parecem estar mais sujeitos a desenvolver DAC cães de raça, especialmente Poodle e do sexo feminino. Além disso, é possível que a pan-histerectomia das fêmeas portadoras auxilie no controle do quadro clínico das pacientes.

Considera-se que os critérios de Favrot podem ser aplicados para a triagem dos pacientes com suspeita de dermatite atópica canina atendidos no HUVET-UFF.

GROSS T.L.; IHRKE P.J.; WALDER E.J.; AFFOLTER V.K. Doenças Perivasculares da Derme. In: Doenças de pele do cão e do gato: diagnóstico clínico e histopatológico. 2.ed. São Paulo: Roca, 2009, p. 194-230.

HENSEL, P.; SANTORO, D.; FAVROT, C.; HILL, P.; GRIFFIN, C. Canine atopic dermatitis: detailed guidelines for diagnosis and allergen identification. BMC Veterinary Research, v.11, p.196, 2015.

HILLIER, A.; GRIFFIN, C.E. The ACVD task force of canine atopic dermatitis $(X)$ : Is there a relationship between canine atopic dermatitis and cutaneous adverse food reactions? Veterinary Immunology and Immunopathology, v. 81, p. 227-231, 2002.

MARSELLA, R.; OLIVRY, T. The ACVD task force on canine atopic dermatitis (VII): mediators of cutaneos inflammation. Veterinary Immunology and Immunopathology, v. 81, p. 205-213, 2001.

MEASON-SMITH, C., OLIVRY, T., LAWHON, S.D., HOFFMANN, A.R. Malassezia species dysbiosis in natural and allergen-induced atopic dermatitis in dogs. Medical Mycology, v.0, p.1-10, 2019.

NOG, J., TAMINIAU, B., FALL, P.A., DAUBE, G., FONTAINE, J. Ear canal microbiota - a comparison between healthy dogs and atopic dogs without clinical signs of otitis externa. Veterinary Dermatology, p. 1-8, 2018.

NISHIFUJI, K. Skin barrier and its role in the pathophysiology of atopic dermatitis. In: NOLI,C.; FOSTER, A.; ROSENKRANTZ, W. Veterinary Allergy. John Wiley \& Sons, 2014. p. 42-50.

OLIVRY, T.; DEBOER, D.J.; FAVROT, C.; JACKSON, H.A.; MUELLER, R.S.; NUTTALL, T.; PRÉLAUD, P. Treatment of canine atopic dermatitis. Clinical practice guidelines from the International Task Force on Canine Atopic Dermatitis. Veterinary Dermatology, p.1-16, 2010.

ONOUE A.; KABASHIMA K.; KOBAYASHI M. et al. Induction of eosinophil- and Th2-attracting epidermal chemokines and cutaneous late-phase reaction in tape-stripped skin. Experimental Dermatology. v. 18, p. 1036-1043, 2009. 
PUCHEU-HASTON, C.M.; BIZIKOVA, P.; EISENSCHENK, M.N.C.; SANTORO, D.; NUTTALL, T; MARSELLA, R. Review: The role of antibodies, autoantigens and food allergens in canine atopic dermatites. Veterinary Dermatology, v.1, p.1-11, 2015.

RONDELLI, M.C.H.; PALACIOS JUNIOR, R.J.G.; TRISTAO, A.P.P.A.; ADAMS, F.K.; TINUCCI-COSTA, M. Cães Atópicos: Estudo retrospectivo da resposta clínica a diferentes protocolos terapêuticos. In: CONGRESSO PAULISTA DAS ESPECIALIDADES. São Paulo: 2012.

SANTOS, M.G.O.; SANTOS, M.M. Perfil da dermatite atópica canina em Salvador - Brasil. Pubvet, v.10, n.11, p.839-843, 2016. SCOTT, D.W.; MILLER JUNIOR, W.H.; GRIFFIN, G.E. Doenças Imunológicas da Pele. In: Dermatologia de Pequenos Animais, 5.ed. Rio de Janeiro: Interlivros. p. 449-580, 1996.

SHAW, S.C.; WOOD, J.L.N.; FREEMAN, J. et al. Estimation of heritability of atopic dermatitis in Labrador and Golden Retrievers. American Journal of Veterinary Research, v. 65, p. 1014-1020, 2004.

SOLOMON, S.E.B.; FARIAS, M.R.; PIMPÃO, C.T. Dermatite atópica canina: fisiopatologia e diagnóstico. Revista Acadêmica de Ciências Agrárias e Ambientais, v.10, n.1, p. 21-28, 2012.

SOUZA, T.M., FIGHERA, R. A., SCHMIDT, C., RÉQUIA, A.H., BRUM, J.S., MARTINS, T.B., BARROS, C.S.L. Prevalência das dermatopatias não-tumorais em cães do município de Santa Maria, Rio Grande do Sul (2005-2008). Pesquisa Veterinária Brasileira, v.29, n.2, p.157-162, 2009.
SZEKERES-BARTHO, J., POLGAR, B., KOZMA, N., MIKO, E., PAR, G., SZEREDAY, L., BARAKONYI, A., PALKOVICS, T., PAPP, O., VARGA, P. Progesterone-dependent immunomodulation. Chemical Immunology Allergy, v.89, p.118-125, 2005.

TARPATAKI, N., THURÓCZY, J., FISCHER, N., JACQUENET, S., ROSTAHER, A., RICHARD, C., ZWICKL, L., OLIVRY, T., FAVROT, C. A pilot study of total and allergen-specific IgE serum levels during anestrous, estrous and pregnancy in healthy female dogs. Veterinary Dermatology, p.1-4, 2018.

VASCONCELOS, J.S., SIQUEIRA, R.S., CLEMENTINO, I.J., GAMA, A., ALVES, A., LUCENA, R.B., DANTAS, A.F.M. Frequência das doenças de pele não tumorais em cães no município de João Pessoa, Paraíba, Brasil (2014-2016). Arquivo Brasileiro de Medicina Veterinária e Zootecnia, v.72, n.4, p.11721184, 2020.

ZANON, J.P., GOMES, L.A., CURY, G.M.M., TELES, T.C., BICALHO, A.P.C.V. Dermatite Atópica Canina. Semina: Ciências Agrárias, v.29, n.4, p.905-920, 2008.

ZUR, G.; IHRKE, P.J.; WHITE, S.D.; KASS, P.H. Canine atopic dermatitis: a retrospective study of 266 cases examined at the University of California, Davis, 1992-1998. Part I. Clinical features and allergy testing results. Veterinary Dermatology, v.13, p.89102, 2002. 